\title{
Topological and Transport Properties of Dirac Fermions in Antiferromagnetic Metallic Phase of Iron-Based Superconductors
}

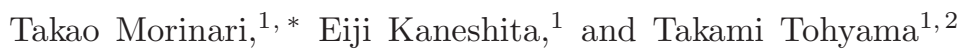 \\ ${ }^{1}$ Yukawa Institute for Theoretical Physics, Kyoto University, Kyoto 606-8502, Japan \\ ${ }^{2}$ JST, Transformative Research-Project on Iron Pnictides (TRIP), Chiyoda, Tokyo 102-0075, Japan
}

(Dated: August 7, 2018)

\begin{abstract}
We investigate Dirac fermions in the antifferomagnetic metallic state of iron-based superconductors. Deriving an effective Hamiltonian for Dirac fermions, we reveal that there exist two Dirac cones carrying the same chirality, contrary to graphene, compensated by a Fermi surface with a quadratic energy dispersion as a consequence of a non-trivial topological property inherent in the band structure. We also find that the presence of the Dirac fermions gives the difference of signchange temperatures between the Hall coefficient and the thermopower. This is consistent with available experimental data.
\end{abstract}

PACS numbers: 73.21.-b, 75.30.Fv, 72.15.Gd, 72.15.Jf

The iron-pnictide high-temperature superconductors have attracted much attention since the discovery [1]. In this multi-orbital system, the Fermi surfaces consist of electron and hole Fermi surfaces of comparable sizes. This multi-band structure leads to the rich physics, presumably including superconductivity with high-transition temperatures [2, 3]. In the parent antiferromagnetic phase, the system remains metallic [4, 5] contrary to the simple antiferromagnetic state in single band systems. This parent antiferromagnetic state possesses a non-trivial topological property; consequently appears a Dirac fermion energy spectrum close to the Fermi energy [6]. In this Letter, we explore the Dirac fermion physics in this system.

Dirac fermions in condensed matter systems were first highlighted by the observation of the anomalous integer quantum Hall effect in graphene [7, 8]. In graphene, the energy spectrum is linear at the corners of the first Brillouin zone, so that the electrons at low energies are well described by the Dirac equation with the speed of light being replaced by the Fermi velocity [9]. Such a unique energy band structure gives rise to several characteristic transport properties [10].

In the iron-pnictide superconductors, a similar linear spectrum was discussed by Ran et al. [6] They pointed out that hybridizations between the Fe $3 d$ orbitals and the pnictide ion $4 p$ orbitals give rise to the band degeneracy characterized by a non-trivial topology. Contrary to a conventional spin density wave (SDW), there are gapless nodal points along the Fermi surface. The topology here is characterized by "vorticity" quantum number, which is associated with the phase winding defined through a two-component spinor wave function. The Fermi surfaces connected by the SDW wave vector have a vorticity zero and a vorticity two: This vorticity mismatch leads to a nodal SDW, and creates Dirac cones near the Fermi

${ }^{*}$ Electronic address: morinari@yukawa.kyoto-u.ac.jp energy.

In this Letter we shall derive the effective theory describing the Dirac fermions based on the five-band model [3] with the SDW mean field analysis [6, 11]. We reveal that there exist two Dirac cones carrying the same chirality contrary to the Dirac fermions in graphene. This unusual feature is due to the presence of another Fermi surface, which does not have a linear spectrum but has chirality. We demonstrate that the scattering rate difference between Dirac fermions and conventional electrons leads to anomalous temperature dependence of the transport coefficients which are consistent with the experiment 12].

For the band structure calculation, we take the five band model

$$
\begin{aligned}
\mathcal{H}_{0} & =\sum_{i, j} \sum_{\mu, \nu} \sum_{s}\left(t_{i \mu, j \nu}+\delta_{i j} \delta_{\mu \nu} \varepsilon_{\mu}\right) d_{i \mu s}^{\dagger} d_{j \nu s} \\
& =\sum_{\mathbf{k}, \mu, \nu, s} \varepsilon_{\mathbf{k}}^{\mu \nu} d_{\mathbf{k} \mu s}^{\dagger} d_{\mathbf{k} \nu s},
\end{aligned}
$$

where $d_{j \mu s}^{\dagger}$ creates an electron with spin $s$ on the $\mu$-th orbital at site $j$. The parameters $t_{i \mu, j \nu}$ and $\varepsilon_{\mu}$ are given in Ref. [3]. Following Ref. [6] we take the following interaction form:

$$
\begin{aligned}
\mathcal{H}_{I}= & U \sum_{j, \mu} n_{j \mu \uparrow} n_{j \mu \downarrow}+(U-2 J) \sum_{j, \mu<\nu, s, s^{\prime}} n_{j \mu s} n_{j \nu s^{\prime}} \\
& +J \sum_{j, \mu<\nu, s, s^{\prime}} d_{j \mu s}^{\dagger} d_{j \nu s^{\prime}}^{\dagger} d_{j \mu s^{\prime}} d_{j \nu s} \\
& +J \sum_{j, \mu<\nu}\left(d_{j \mu \uparrow}^{\dagger} d_{j \mu \downarrow}^{\dagger} d_{j \nu \downarrow} d_{j \nu \uparrow}+\text { H.c. }\right)
\end{aligned}
$$

where $n_{j \mu s}=d_{j \mu s}^{\dagger} d_{j \mu s}^{\dagger}$. The mean field Hamiltonian for the SDW state reads,

$$
\mathcal{H}_{M F}=\sum_{\mathbf{k} \in R B Z, \mu, \nu, s}\left(d_{\mathbf{k} \mu s}^{\dagger} d_{\mathbf{k}+\mathbf{Q}, \mu s}^{\dagger}\right)\left[\mathcal{H}_{\mathbf{k} s}\right]_{\mu \nu}\left(\begin{array}{c}
d_{\mathbf{k} \nu s} \\
d_{\mathbf{k}+\mathbf{Q}, \nu s}
\end{array}\right),
$$




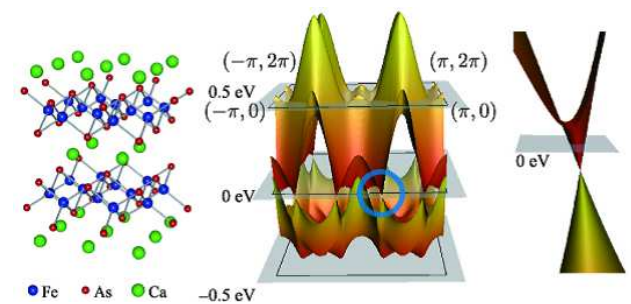

FIG. 1: (color online) (Left) The crystal structure of $\mathrm{CaFe}_{2} \mathrm{As}_{2}$. (Middle) The electron band structure near the Fermi energy in the SDW state. The unit cell is taken by the square lattice formed by Fe atoms. (Right) The magnification of the band structure near the Dirac point indicated by the circle in the left.

where $\mathbf{Q}=(\pi, 0)$ and the $\mathbf{k}$ summation is taken over the reduced Brillouin zone. The matrix $\mathcal{H}_{\mathbf{k} s}$ is given by

$$
\left[\mathcal{H}_{\mathbf{k} s}\right]_{\mu \nu}=\left(\begin{array}{cc}
\varepsilon_{\mathbf{k}}^{\mu \nu} & \pm m_{S D W}^{\mu \nu} \\
\pm m_{S D W}^{\mu \nu} & \varepsilon_{\mathbf{k}+\mathbf{Q}}^{\mu \nu}
\end{array}\right)
$$

where we take the plus (minus) sign for $s=\uparrow(\downarrow)$. The mean field order parameters, $m_{S D W}^{\mu \nu}$, are determined by solving the mean field equations in a self-consistent way. Figure 1 shows the electron band structure calculated in the SDW state with $U=1.2 \mathrm{eV}$ and $J=0.25 \mathrm{eV}$ with the zero temperature magnetic moment $1.0 \mu_{B}$, which is close to the value observed by neutron scattering in $\mathrm{BaFe}_{2} \mathrm{As}_{2}$ [13].

In the SDW state, Dirac points appear near the Fermi energy. Those Dirac points exist on the $k_{x}$ axis and, in some parameter region, on the $k_{y}$ axis as well but not at the high symmetric points in the Brillouin zone. Since the Dirac points appearing on the $k_{y}$ axis are not protected by topology [6], we focus on the Dirac points on the $k_{x}$ axis. These Dirac points are stable as long as the magnetic moment is less than $3 \mu_{B}$.

In order to investigate the physical properties of the Dirac fermions we derive the effective Hamiltonian around the Dirac point following Ref. 14], in which the Dirac fermions in the organic conductor $\alpha$-(BEDT$\mathrm{TTF})_{2} \mathrm{I}_{3}$ were analyzed. The effective Hamiltonian is used to determine chirality and the Landau level structure of Dirac fermions.

First we expand the Hamiltonian $\mathcal{H}_{\mathbf{k}}$ around the Dirac point $\mathbf{k}_{c}$ with denoting $\mathbf{k}=\mathbf{k}_{c}+\delta \mathbf{k}$,

$$
\mathcal{H}_{\mathbf{k}}=\mathcal{H}_{\mathbf{k}_{c}}+\frac{\partial \mathcal{H}_{\mathbf{k}_{c}}}{\partial k_{x}} \delta k_{x}+\frac{\partial \mathcal{H}_{\mathbf{k}_{c}}}{\partial k_{y}} \delta k_{y}+\ldots
$$

(The spin index is suppressed hereafter.) Next, noting that a Dirac point is formed by two eigenstates we construct the effective $2 \times 2$ Hamiltonian using those two eigenstates denoted by $|\mathbf{k}, \pm\rangle$. The general form of the effective Hamiltonian is [14],

$$
\mathcal{H}_{\delta \mathbf{k}}^{e f f}=\sum_{\rho=0, x, y, z} \delta \mathbf{k} \cdot \mathbf{v}_{\rho} \sigma_{\rho}
$$

where $\boldsymbol{\sigma}=\left(\sigma_{x}, \sigma_{y}, \sigma_{z}\right)$ are the Pauli matrices and $\sigma_{0}$ is the unit matrix. (We set $\hbar=1$.) The parameters $\mathbf{v}_{\rho}$ are determined by the following equation with $\alpha=x, y$ :

$$
\left\langle\mathbf{k}, s\left|\frac{\partial \mathcal{H}_{\mathbf{k}_{c}}}{\partial k_{\alpha}}\right| \mathbf{k}, s^{\prime}\right\rangle=\sum_{\rho=0, x, y, z} v_{\rho}^{\alpha}\left(\sigma_{\rho}\right)_{s s^{\prime}} .
$$

Figure 2(a) shows the energy band dispersion of the state (shown in Fig. 10 along the $k_{x}$ axis. The Dirac points are at $\mathbf{k}_{c}=( \pm 0.829,0)$. The positions of the Dirac points are close to the values reported in angle resolved photoemission spectroscopy measurements [15]. The effective Hamiltonian is given by

$$
\mathcal{H}_{\mathbf{k}}=\left(E_{c} \pm v_{0}^{x} k_{x}\right) \sigma_{0} \pm\left(v_{z}^{x} k_{x} \sigma_{z}+v_{x}^{y} k_{y} \sigma_{x}\right),
$$

where $E_{c}-E_{F}=-20 \mathrm{meV}, v_{0}^{x} / a=0.286 \mathrm{eV}, v_{x}^{y} / a=$ $0.229 \mathrm{eV}, v_{z}^{x} / a=0.672 \mathrm{eV}$ with $a$ the lattice constant. The non-zero value of $v_{0}^{x}$ implies that the Dirac cone is tilted in the $k_{x}$ direction. As a result, we expect a strong anisotropy in the Fermi velocity.

Now we discuss chirality associated with Dirac fermions. In Fig. 2(b) and (d), the vector $\mathbf{n}_{\mathbf{k}} \equiv$ $\langle\mathbf{k},+|\boldsymbol{\sigma}| \mathbf{k},+\rangle$ is shown around the two Dirac points. The vector field $\mathbf{n}_{\mathbf{k}}$ shows a vortex configuration with vorticity one. A remarkable fact is that the two Dirac cones have the same vorticity: The vector $\mathbf{n}_{\mathbf{k}}$ is rotated clockwise when one goes around each Dirac point. In conventional Dirac fermion systems, like graphene, two Dirac cones have the opposite chirality by symmetry. The same is true for the Dirac cones in $\alpha$-(BEDT$\mathrm{TTF})_{2} \mathrm{I}_{3}$. This unusual property is understood by considering the chirality associated with the hole band around $\Gamma$ point as shown in Fig. 2(c). Although the energy dispersion around the $\Gamma$ point is quadratic, and an energy gap lies between two relevant bands, we can apply the above analysis in constructing the effective Hamiltonian: In the quadratic dispersion case, the parameters $\mathbf{v}_{\rho}$ are linear functions with respect to $k_{x}$ or $k_{y}$ with higher order corrections. Along the circle represented by $k=\sqrt{k_{x}^{2}+k_{y}^{2}}=0.1$, we find $\mathcal{H}_{\mathbf{k}}=a_{\mathbf{k}} \sigma_{0}+b_{\mathbf{k}} \sigma_{z}+c_{\mathbf{k}} \sigma_{x}$, with $a_{\mathbf{k}}=-0.508 k_{x}^{2}-0.475 k_{y}^{2}, b_{\mathbf{k}}=-0.191 k_{x}^{2}+2.56 k_{y}^{2}$, and $c_{\mathbf{k}}=2.80 k_{x} k_{y}$. The winding is described by the following vector: $\left(n_{x}, n_{z}\right)=\left(c_{k}, b_{k}\right) / \sqrt{b_{k}^{2}+c_{k}^{2}}$. As shown in Fig. 2(c), the vector $\mathbf{n}_{\mathbf{k}}$ rotates counter-clockwise twice when one goes around the $\Gamma$ point. This chirality exactly compensates chirality of the two Dirac cones. We note that the scattering between the two Dirac cones, which is still under debate in graphene [16], is qualitatively different from that in graphene because these Dirac cones carry the same chirality. It is worth of pointing out that this chirality property is compatible with the interband Cooper pairing between the hole band around the $\Gamma$ point and the Dirac cones.

Now we turn to the physical properties associated with the Dirac fermions. One of the most significant effects associated with the Dirac fermion chirality is the suppression of backward scattering [17]. As a consequence the 

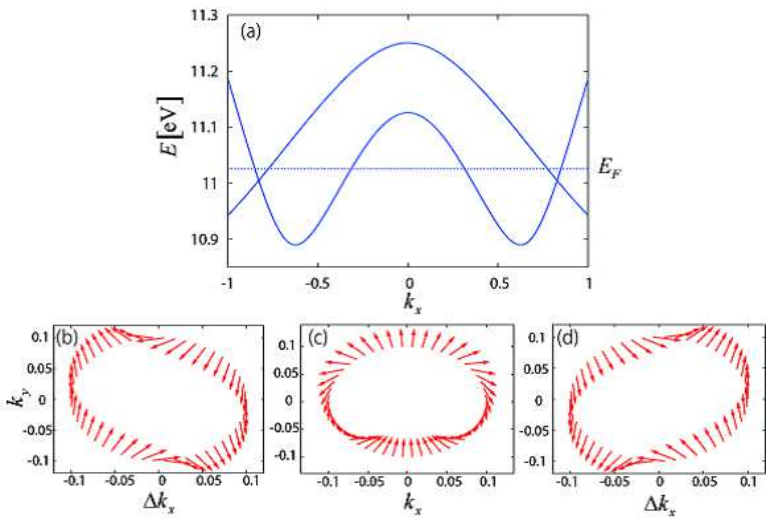

FIG. 2: (color online) (a) The energy bands along the $k_{x}$ axis. The dotted line represents the Fermi energy. Two Dirac points are located at $( \pm 0.829,0)$. The direction of the vector $\mathbf{n}(\mathbf{k})=\left(n_{x}(\mathbf{k}), n_{z}(\mathbf{k})\right)$ around $(\mathrm{a})(-0.829,0),(\mathrm{b})(0,0)$, and (c) $(0.829,0)$.

scattering ratio is different between Dirac fermions and conventional electrons. Reflecting this difference some transport coefficients clearly exhibit contributions from Dirac fermions [18] even if the Dirac fermions are the minor carrier [19].

We consider a phenomenological two-band model consisting of a hole band, with a conventional energy spectrum, and an electron band, with the Dirac fermion energy spectrum. The charge current is given by $\mathbf{j}=$ $\sigma \mathbf{E}+\alpha(-\nabla T)$, where $\sigma$ and $\alpha$ are tensors computed by the Jones-Zener solution of the Boltzmann equation [20]. The contribution from the Dirac fermions is denoted by $\sigma^{(e)}$ and $\alpha^{(e)}$ and that from the holes is denoted by $\sigma^{(h)}$ and $\alpha^{(h)}$. The sign change of the Hall coefficient $R_{H}=\sigma_{x y} /\left(B_{z} \sigma_{x x}^{2}\right)$ with $B_{z}$ the applied magnetic field in the $z$ direction occurs at $\sigma_{x y}^{(e)} / \sigma_{x y}^{(h)}=1$. On the other hand, the sign change in the thermopower occurs when $\alpha_{x x}^{(e)} / \alpha_{x x}^{(h)}=1$. For the energy dispersions, we take $\varepsilon_{k}^{(e)}=v k-\varepsilon_{e}$ and $\varepsilon_{k}^{(h)}=-k^{2} /(2 m)+\varepsilon_{h}$ with energy levels $\epsilon_{e}$ and $\epsilon_{h}$. At finite temperatures, electrons may be scattered by phonons and spin waves. Here we focus on temperatures lower than the characteristic temperatures associated with those excitations and assume constant scattering times, $\tau_{e, h}$. In order to reduce the number of parameters, we set $m v^{2} / 2=\varepsilon_{e} \equiv \varepsilon_{0}$. The difference between $\tau_{e}$ and $\tau_{h}$ is parameterized by $\tau_{r} \equiv \tau_{h} / \tau_{e}$. In Fig. 31(a) and (b) we show $\sqrt{r_{e} / r_{h}} \equiv \sqrt{\tau_{h}^{2} \sigma_{x y}^{(e)} /\left(\tau_{e}^{2} \sigma_{x y}^{(h)}\right)}$ and $s_{e} / s_{h} \equiv \tau_{h} \alpha_{x x}^{(e)} /\left(\tau_{e} \alpha_{x x}^{(h)}\right)$ for differnt values of $n_{r} \equiv$ $n_{e} / n_{h}$ with $n_{e(h)}$ the electron (hole) density. Whether there are sign changes or not depends on $\tau_{r}$ and $n_{r}$. (Note that $n_{r}$ is controlled by changing $\varepsilon_{h}$.) The sign change occurs when $\tau_{r}=\sqrt{r_{e} / r_{h}}, s_{e} / s_{h}$. In general, the sign change occurs at different temperatures for the Hall coefficient and the thermopower because of their different dependences on $\tau_{r}$.
We show the Seebeck coefficient, the Hall coefficient, and the Nernst coefficient, $\nu=\left(\alpha_{x x} \sigma_{x y}-\right.$ $\left.\alpha_{x y} \sigma_{x x}\right) /\left[B_{z}\left(\sigma_{x x}^{2}+\sigma_{x y}^{2}\right)\right]$, in Fig. 3 $3\left(\right.$ c) for $\tau_{r}=0.45$ and $n_{r}=0.05$. The result is consistent with the experiment reported in Ref. [12], where the similar sign changes were observed in $\mathrm{CaFe}_{2} \mathrm{As}_{2}$. Note that we expect large contributions from the Dirac fermions for the Nernst coefficient as well because the quantity is quadratically dependent on the scattering time. We point out that a naive application of the Jones-Zener solution to the SDW mean field state with the same scattering time for all bands does not lead to these temperature dependence even if we take into account the temperature dependence of the order parameter. In $\mathrm{CaFe}_{2} \mathrm{As}_{2}$, the energy dispersion in the $k_{z}$ direction may not be negligible. Therefore, we assumed here a moderate value for $\tau_{r}$. For systems with strong twodimensionality, like the so-called 1111 system, we expect a small $\tau_{r}$. In such a case Dirac fermions markedly contribute to the transport coefficients.

If we assume a conventional energy dispersion for the electron band, the temperature dependence of $\sqrt{r_{e} / r_{h}}$ is qualitatively different from the Dirac fermion case as shown in the inset in Fig. 3(a). In this case, the Hall coefficient behaves qualitatively different from Fig. 3(c). The Hall coefficient is positive at low temperatures, unlike the Dirac fermion case.

A systematic change of these sign changes are expected by controlling the electron density. In Fig. 4 we show the doping dependence of the Dirac point and its position in the Brillouin zone in the five-band SDW state. Both decrease monotonically by increasing electron density. Interestingly the Dirac point crosses the Fermi energy at the hole doping side.

Now we propose an experiment to directly observe the evidence of the Dirac fermions. In conventional electrons, the Landau level energies are equally separated. By contrast, the Dirac fermion Landau level energies are not. For the tilted Dirac cone (87), the Landau level energy spectrum is given by [21]

$$
E_{n}=\operatorname{sgn}(n) \frac{\sqrt{v_{z}^{x} v_{x}^{y}}}{\ell_{B}} \sqrt{2 \lambda^{3}|n|},
$$

with $\ell_{B}=1 / \sqrt{e B}$ the magnetic length and $\lambda=$ $\sqrt{1-\left(v_{0}^{x} / v_{z}^{x}\right)^{2}}$. This Dirac fermion Landau level structure can be observed by scanning tunneling spectroscopy (STS) measurements. At graphite surfaces under magnetic fields a series of peaks in the tunnel spectra associated with Landau quantization of the quasi-twodimensional electrons were observed [22, 23]. The observed Landau levels are in good agreement with those expected for a surface layer, graphene. From our effective Hamiltonian, we evaluated that $E_{1} \simeq 10 \mathrm{meV}$ at $B=10 \mathrm{~T}$ while $E_{1} \simeq 130 \mathrm{meV}$ in graphene. Using STS, we expect that the Dirac fermion Landau level structure should be observed in the parent antiferromagnetic state.

To conclude, we have investigated the Dirac fermions in the parent antiferromagnetic state of the iron-based 

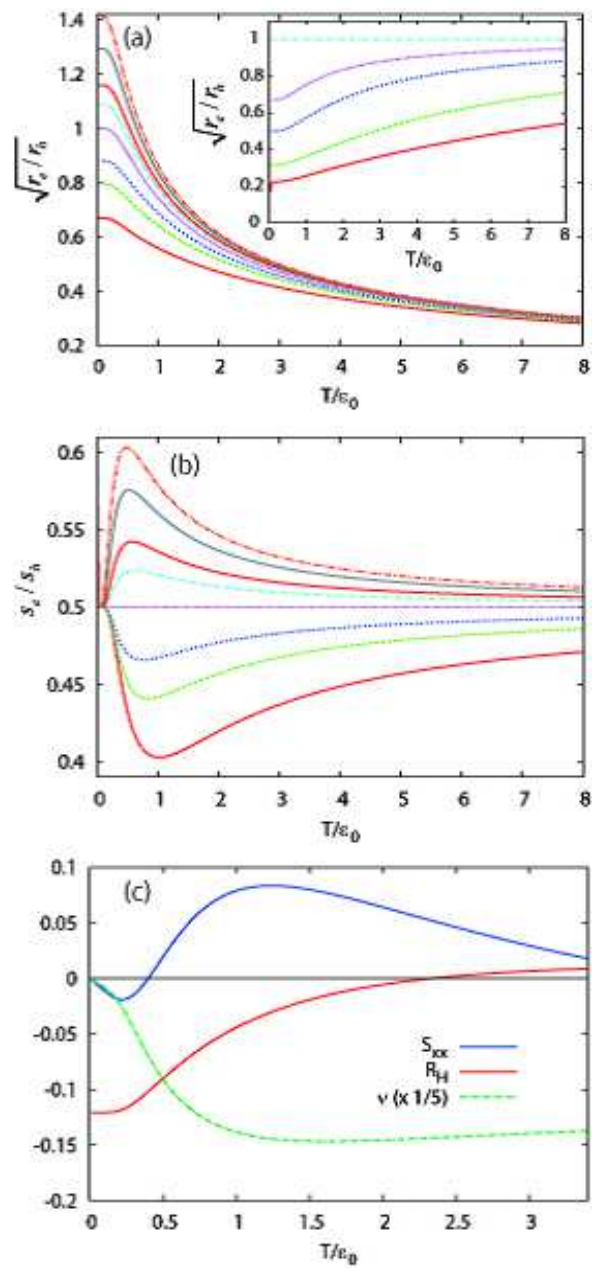

FIG. 3: (color online) The temperature dependence of the ratios $\sqrt{r_{e} / r_{h}}$ (a) and $s_{e} / s_{h}$ (b) for different values of the density ratio $n_{r} . \quad\left(n_{r}=1.0,0.70,0.45,0.35,0.25,0.15,0.10,0.05\right.$ from the top to the bottom.) The inset in (a) shows the temperature dependence of $\sqrt{r_{e} / r_{h}}$ for conventional energy dispersions. $\left(n_{r}=1.0,0.45,0.25,0.10,0.05\right.$ from the top to the bottom.) (c) The temperature dependence of the Hall coefficient, the Seebeck coefficient, and the Nernst coefficient, which are normalized by $\left(2 \pi a^{2} /|e|\right)\left(\hbar v / \varepsilon_{0} a\right)^{2}, k_{B} /|e|$, and $\left(k_{B} a^{2} /(2 \pi \hbar)\right)\left(\hbar v / \varepsilon_{0} a\right)\left(v \tau_{D} / a\right)$, respectively, with $k_{B}$ the Boltzmann constant. superconductors. There exist two tilted Dirac cones with the same chirality contrary to the Dirac cones in graphene. The sign changes observed experimentally in the thermopower and the Hall coefficient are consistently understood from the Dirac fermion picture. We propose that the Dirac fermion Landau level structure be observed by STS measurements. Since the two Dirac cones carry the same chirality, the intervalley scattering is qualitatively different from ordinary Dirac fermion systems. Compared to graphene, the valley splitting occurs in a different manner that would be clarified in the magnetoresistance.

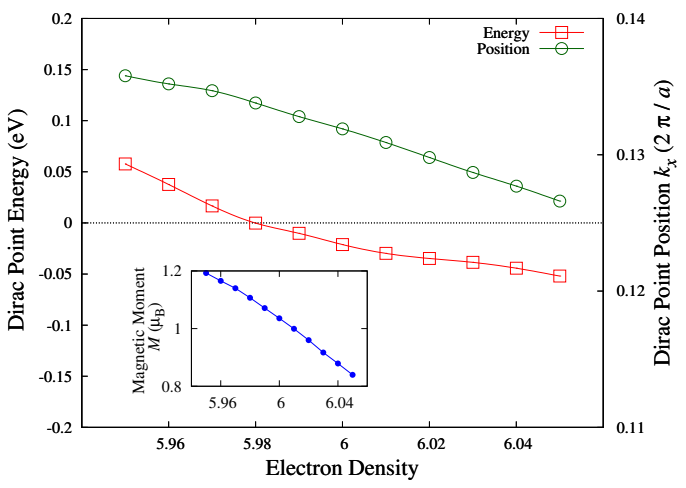

FIG. 4: (color online) The electron density dependence of the Dirac point and the Dirac point position in the Brillouin zone denoted by $\left(k_{x}, 0\right)$. The inset shows the electron density dependence of the magnetic moment.

This work was supported by the Grant-in-Aid for Scientific Research from the Ministry of Education, Culture, Sports, Science and Technology of Japan; the Global COE Program "The Next Generation of Physics, Spun from University and Emergence"; and Yukawa Institutional Program for Quark-Hadron Science at YITP. Numerical computation in this work was carried out at the Yukawa Institute Computer Facility. E.K. is the Yukawa Fellow and this work was partially supported by Yukawa Memorial Foundation.
[1] Y. Kamihara, T. Watanabe, M. Hirano, and H. Hosono, J. Am. Chem. Soc. 130, 3296 (2008).

[2] I. I. Mazin, D. J. Singh, M. D. Johannes, and M. H. Du, Phys. Rev. Lett. 101, 057003 (2008).

[3] K. Kuroki, S. Onari, R. Arita, H. Usui, Y. Tanaka, H. Kontani, and H. Aoki, Phys. Rev. Lett. 101, 087004 (2008).

[4] S. Sebastian, J. Gillett, N. Harrison, P. Lau, D. Singh, C. Mielke, and G. Lonzarich, J. Phys.: Condens. Matter 20, 422203 (2008).

[5] J. G. Analytis, R. D. McDonald, J.-H. Chu, S. C. Riggs,
A. F. Bangura, C. Kucharczyk, M. Johannes, and I. R. Fisher, Phys. Rev. B 80, 064507 (2009).

[6] Y. Ran, F. Wang, H. Zhai, A. Vishwanath, and D.-H. Lee, Phys. Rev. B 79, 014505 (2009).

[7] K. S. Novoselov, A. K. Geim, S. V. Morozov, D. Jiang, M. I. Katsnelson, I. V. Grigorieva, S. V. Dubonos, and A. A. Firsov, Nature 438, 197 (2005).

[8] Y. B. Zhang, Y. W. Tan, H. L. Stormer, and P. Kim, Nature 438, 201 (2005).

[9] P. Wallace, Phys. Rev. 71, 622 (1947).

[10] A. K. Geim and K. S. Novoselov, Nat. Mater. 6, 183 
(2007).

[11] E. Kaneshita, T. Morinari, and T. Tohyama, Phys. Rev. Lett. 103, 247202 (2009).

[12] M. Matusiak, Z. Bukowski, and J. Karpinski, Phys. Rev. B 81, 020510(R) (2010).

[13] Q. Huang, Y. Qiu, W. Bao, M. A. Green, J. W. Lynn, Y. C. Gasparovic, T. Wu, G. Wu, and X. H. Chen, Phys. Rev. Lett. 101, 257003 (2008).

[14] A. Kobayashi, S. Katayama, Y. Suzumura, and H. Fukuyama, J. Phys. Soc. Jpn. 76, 034711 (2007).

[15] P. Richard, K. Nakayama, T. Sato, M. Neupane, Y.-M. Xu, J. Bowen, G. Chen, J. Luo, N. Wang, H. Ding, et al., arXiv:0909.0574 (2009).

[16] Z. Jiang, Y. Zhang, T.-W. Tan, H. Stormer, and P. Kim, Solid State Commun. 143, 14 (2007).
[17] T. Ando, T. Nakanishi, and R. Saito, J. Phys. Soc. Jpn. 67, 2857 (1998).

[18] H. Fukuyama, JPSJ Online-News and Comments May 12 (2008).

[19] N. Harrison and S. E. Sebastian, arXiv:0910.4199 (2009).

[20] J. Ziman, Electrons and phonons: the theory of transport phenomena in solids (Oxford University Press, USA, 2001).

[21] T. Morinari, T. Himura, and T. Tohyama, J. Phys. Soc. Jpn. 78, 023704 (2009).

[22] T. Matsui, H. Kambara, Y. Niimi, K. Tagami, M. Tsukada, and H. Fukuyama, Phys. Rev. Lett. 94, 226403 (2005).

[23] G. Li and E. Andrei, Nature Phys. 3, 623 (2007). 